\title{
Examination of clonal evolution in chronic lymphocytic leukemia
}

\author{
Dorota Koczkodaj $^{1}$ (D) Sylwia Popek-Marciniec ${ }^{1} \cdot$ Szymon Zmorzyński $^{1} \cdot$ Ewa Wąsik-Szczepanek $^{2} \cdot$ Agata A. Filip $^{1}$
}

Received: 30 May 2019 / Accepted: 20 July 2019 / Published online: 2 August 2019

(c) The Author(s) 2019

\begin{abstract}
Chronic lymphocytic leukemia (CLL) is one of the most frequent lymphoproliferative diseases. CLL is characterized by unusual heterogeneity, which probably reflects its biological and genetic lack of homogeneity. Clonal chromosome aberrations belong to the most important prognostic and predictive factors in CLL. This research was aimed at observing clonal evolution in CLL at the chromosomal level, and assessing its clinical significance in relation to selected prognostic factors. The study involved 72 untreated patients with CLL. The preliminary investigations using cytogenetic banding analysis (CBA) and FISH were performed at the time of diagnosis, and again after about 24 months to observe clonal changes (clonal evolution). In addition, other parameters were evaluated, i.e., the expression of ZAP-70 kinase, CD38 antigen, and the mutation statuses of IGVH and NOTCHI genes. Classic prognostic factors, i.e., categorized ZAP70 and CD38 expressions as well as mutations in $I G V H$ and $N O T C H I$ genes did not influence the course of clonal evolution in the examined group of patients. Clonal evolution was detected in $45.8 \%$ of patients by means of CBA, and in $19.4 \%$ patients with FISH. Analysis of chromosomal aberrations in the examined group of patients showed that the incidence of $17 \mathrm{p}$ deletions and translocations in karyotypes has a negative impact on overall survival. CE was found to be a risk factor for the occurrence of disease progression $(\mathrm{OR}=2.22)$. Our observations indicate that combined CBA and FISH are the most optimal techniques for monitoring clonal evolution in the course of CLL.
\end{abstract}

Keywords Chronic lymphocytic leukemia $\cdot$ Clonal evolution $\cdot$ Cytogenetic banding analysis $\cdot$ FISH

\section{Introduction}

Chronic lymphocytic leukemia (CLL) is one of the most common lymphoproliferative diseases that occur in elderly people in Europe and North America. It is a monoclonal disease, characterized by accumulation of small, morphologically mature, resting lymphocytes B in peripheral blood, bone marrow, lymph nodes, and spleen. Leukemic lymphocytes are characterized by a unique immunophenotype, which involves coexpressions of the surface antigens: CD19+, CD20+, CD5+i, and CD23+; and poor expressions of the IgM and IgD immunoglobulins. CLL cells may

Dorota Koczkodaj

dorota.koczkodaj@umlub.pl

1 Department of Cancer Genetics with the Cytogenetic Laboratory, Medical University of Lublin, Ul.

Radziwiłłowska 11, 20-080 Lublin, Poland

2 Department of Hematooncology and Bone Marrow Transplantation, Medical University of Lublin, Lublin, Poland show differentiated expressions of CD38, CD25, and CD79b antigens on the surface and ZAP-70 kinase in the cytoplasm [1-4].

CLL belongs to the class of heterogenous diseases. There is a group of patients with benign, slow course of disease, which does not require treatment, and another comprising patients with rapid progression despite the use of aggressive therapy. There is also a group of patients with a moderate prognosis. Patients from these prognostic groups differ as to the mutation status of $I G V H$ gene, the expression profile of the genes that encode the proteins ZAP70, LPL, and ADAM29, and expression of the CD38 antigen. In addition, mutations in the NOTCH1 gene were observed in patients with CLL. Mutations in the PEST domain of this gene are an unfavorable prognostic factor. There is also a high risk of CLL transformation in the Richter syndrome in patients with such a mutation [5].

Genetic alterations which include clonal chromosome aberrations also play an important role.

Commonly used methods for detecting chromosome aberrations accompanying the expansion of a cell clone 
Table 1 Clinical and laboratory characteristics of CLL patients

\begin{tabular}{llll}
\hline $\begin{array}{l}\text { Parameter } \\
\text { median (range) }\end{array}$ & Whole tested group $(N=72)$ & Women $(N=34)$ & Men $(N=38)$ \\
\hline Age & $64.00(36.00-86.0)$ & $63.00(36.00-78.00)$ & $64.50(38.00-86.00)$ \\
HGB (g/dL) & $13.10(5.90-18.10)$ & $13.20(5.90-16.60)$ & $12.85(8.00-18.10)$ \\
WBC (G/L) & $57.75(6.90-628.00)$ & $53.20(6.90-149.00)$ & $58.25(9.70-628.00)$ \\
LIMF (G/L) & $51.55(5.70-540.00)$ & $43.15(5.70-131.80)$ & $52.65(6.01-540.00)$ \\
PLT (G/L) & $173.00(47.00-341.00)$ & $202.50(84.00-341.00)$ & $155.50(47.00-325.00)$ \\
B2 M (mg/L) & $3.31(0.80-4434.50)$ & $3.19(1.86-4421.90)$ & $3.56(0.80-4434.50)$ \\
LDH (U/L) & $351.00(108.00-1732.00)$ & $356.00(108.00-997.00)$ & $327.50(151.00-1732.00)$ \\
CD38 (\%) & $13.37(0.15-82.36)$ & $20.13(0.15-54.00)$ & $25.51(0.54-82.36)$ \\
ZAP70 (\%) & $18.45(1.51-69.74)$ & $21.74(1.51-68.58)$ & $23.77(2.17-69.14)$ \\
TTM & $10.40(2.40-25.20)$ & $8.60(2.40-12.40)$ & $11.35(2.80-25.20)$ \\
\hline
\end{tabular}

TTM Score total tumor mass score are the cytogenetic analysis of metaphase cells by banding (CBA) or the molecular techniques such as FISH used to metaphase chromosomes or interphase nuclei.

The studies using methods of classical cytogenetics have allowed karyotype lesions of a clonal character to be observed in 16-43\% of patients with CLL [4, 6]. Unfortunately, in the study of clonal character, the use of methods of classical cytogenetics is associated with multiple restrictions, which are mainly due to the low mitotic index of leukemic cells in cultures in vitro. Thanks to the use of new mitogens in in vitro cultures, the efficiencies of the methods of classical cytogenetics and the assessments of karyotype in CLL patients have been increased.

At present, the reference method for detecting chromosomal changes is FISH. This technique enables an effective assessment of anomalies in more than $83 \%$ of CLL patients [7-9].

Detected genome alterations, including clonal chromosomal aberrations, are independent prognostic factors that enable identification of patients with CLL of various clinical course, time to first-line treatment, and overall survival.

The most frequently detected chromosomal aberrations in CLL by means of these research methods include deletions or translocations of chromosomes 13, 11, 14, 17, and 6 in regions 13q, 11q, 14q, 17p, and 6q, respectively, and trisomy of chromosome 12 [10].

The phenomenon of clonal evolution (acquisition of chromosomal abnormalities during the course of the disease) that affects the clinical course of CLL is a subject of increasing attention. Our research focused on the observation of clonal evolution and its clinical significance in the course of CLL in relation to selected prognostic factors.

\section{Methods}

The material for the research were peripheral blood lymphocytes from 72 individual subjects with chronic lymphocytic leukemia hospitalized over the years 2005-2015 at the Department and Clinic of Hematooncology and Bone Marrow Transplantation, Medical University, Lublin, Poland.

Informed consent of patients for blood collection and positive opinion of the Bioethics Committee at the Medical University in Lublin (no. KE-0254/153/2010) were obtained.

Patients with CLL were diagnosed according to standard morphological and immunophenotypic criteria [11]. The test group comprised 34 women and 38 men aged 36 to 86 years (on average $63.22 \pm 10.42$ ). The patients were at different stages of disease according to the classification by Rai et al. 20 patients (28\%) was at Rai stage 0 , the group of 13 patients (18\%) at stage I, 29 patients (40.2\%) at stage II, 7 patients (9.7\%) at stage III while 3 people $(4.1 \%)$ at the fourth stage of disease progression.

The clinical and laboratory characteristics of the CLL patients are shown in Table 1.

Cytogenetic analyses (classical cytogenetic tests and FISH) were performed in all patients twice (baseline and follow-up) at the time of diagnosis and prior to possible start of treatment, and repeated after approx. 24 months.

For all the subjects, immunophenotyping was performed according to standard procedures using the FACSCalibur II flow cytometer from Becton-Dickinson, USA. The analysis of the results was obtained by the CellQuest Pro program (Becton-Dickinson); 10,000 cells for each sample were assessed after incubation with monoclonal mouse anti-human CD5-PE, CD19-PE-Cy5, CD23-FITC, CD38FITC, and ZAP70 antibodies along with appropriate isotype controls (all from BD Bioscience). Antibodies were applied at $1 \mu \mathrm{g} / 100 \mu \mathrm{L}$ of cell suspension $\left(1 \times 10^{6}\right.$ cells in $1 \% \mathrm{BSA} / \mathrm{PBS}$ ), and samples were processed according to 
the manufacturer's instructions. The expression of the CD38 surface antigen and ZAP-70 has been categorised according to existing standards, i.e., high expression for CD38 (>30\%) and high expression for ZAP-70 (>20\%) [11].

Genomic DNA was extracted from $200 \mu \mathrm{L}$ peripheral blood samples collected in EDTA with GeneMATRIX Quick Blood DNA Purification Kit (EURx, Poland), according to the manufacturer's manual. DNA quality and quantity were assessed by means of NanoDrop 200 spectrophotometer (ThermoScientific) and by agarose gel electrophoresis. For further investigation, samples containing nondegraded, high molecular weight DNA at the concentration of at least $100 \mathrm{ng} / \mu \mathrm{L}$, with A260/A280 ratio between 1.8 and 2.0, were used.

The most common NOTCH1 mutations located in fragment of exon 34 were analyzed by direct sequencing. DNA was amplified with high fidelity Advantage HD polymerase (Clontech). The assessment of mutation state of the NOTCH1 gene was performed using the sequencing technique using an ABI Prism 3130 Genetic Analyzer (Applied Biosystems) [12]. All primers and conditions were designed at the Department of Cancer Genetics with the Cytogenetic Laboratory, Medical University of Lublin (sequences and detailed conditions are available on request).

Testing of rearrangement and somatic hypermutation of the VDJ genes of the immunoglobulin heavy chain was performed based on the sequencing technique. The results of $I G V H$ genes sequencing were compared with the germ line sequences, available in the Immunogenetics (IMGT) database, using the IMGT/V-QUEST software. The analysis identified the $V D J$ genes occurring in the rearrangement examined, the number and type of mutations present therein, and determined whether the rearrangement is of a functional nature. Sequences having identity of $\geq 98 \%$ with the germ sequence have been considered to be unmutated and those having $<98 \%$ have been deemed mutated.

\section{Cytogenetic analyses}

The cultures were run in a standard manner in sterile Falcon (Sarstedt) vessels on the RPMI 1640 medium with L-glutamine with $15 \%$ inactivated fetal calf serum (FCS) vol/vol (Biomed), $100 \mathrm{U} / \mathrm{mL}$ penicillin G sodium salt, $100 \mu \mathrm{g} / \mathrm{mL}$ of streptomycin sulfate, and $0.25 \mu \mathrm{g} / \mathrm{mL}$ of amphotericin B (Gibco, Invitrogen). The starting culture concentration was $2 \times 10^{6}$ cells $/ \mathrm{mL}$ of the medium. The cultures were run at $37{ }^{\circ} \mathrm{C}$ in the atmosphere of $5 \% \mathrm{CO}_{2}$.

To obtain a large mitotic index, two versions of the culture were assumed. To both a suitable mix of stimulation factors has been added: in version A-PWM-2,5 $\mu \mathrm{g} /$ $\mathrm{mL}$ (Pokeweed Mitogen, SIGMA Aldrich), TPA-10 ng/mL (12-O-tetradecanoylphorbol 13-acetate; Sigma Aldrich), calcium ionophore-7.5 $\times 10^{-7} \mathrm{~mol} / \mathrm{mL}$ (4-bromo-calcium ionophore; Sigma Aldrich), oligoCpG DSP30-2 $\mu \mathrm{mol} / 1$ (TibMolBiol), in version B-PWM-2.5 $\mu \mathrm{g} / \mathrm{mL}$, TPA-10 ng/ $\mathrm{mL}$, calcium ionophore $7.5 \times 10^{-7} \mathrm{~mol} / \mathrm{mL}$, oligoCpG DSP30-2 $\mu \mathrm{mol} /$, LPS-50 $\mu \mathrm{g} / \mathrm{mL}$ (Escherichia coli lipopolysaccharide; Sigma Aldrich).

The mixtures of stimuli used have been adequately tested and selected for the type of the cell culture concerned [13].

The cultures were run for 3 to 5 days and then terminated in a standard manner. The differential staining of metaphases was conducted in the cytogenetic preparations $(G$ and $R$ bands) and analyzed in a Nikon Upright Microscope eclipse $\mathrm{Ni}-\mathrm{U}$ microscope with the magnification $\times 1000$ under the immersion, lens $100 \times 1.45$ Plan Apo $\lambda$ Oil. The chromosome analysis was carried out using the CytoVision computer system from Applied Imaging and Applied Spectral Imaging Case Data Manager Capture and Analysis V7.1. Cytogenetic changes were described according to ISCN 2016 [14].

\section{FISH}

Selected chromosomal aberrations in interphase nuclei and metaphase chromosomes were assessed using the FISH method with the use of a fluorochrome-marked probe panel. The set of probes used for the study contained the following single probes: LSI D13S319 (13q14) labeled with SpectrumOrange, LSI 13 (RBI) labeled with SpectrumOrange, LSI ATM (11q22.3) labeled with SpectrumOrange, LSI p53 (17p13) SpectrumOrange, SPP 12 labeled with SpectrumGreen or SpectrumOrange, LSI MYB (6q23) labeled with SpectruAqua and the multi-coloured probes: LSI D13S319/ LSI 13q34/CEP12 marked with appropriate SpectrumOrange/SpectrumAqua/SpectrumGreen fluorochromes, LSI p53/LSI ATM probe marked with SpectrumOrange/SpectrumGreen, LSI IGH/CCNDIdual color, dual fusion translocation probe, LSI break apart IGH translocation probe firmy VYSIS- Abbott Molecular. We also used Kreatech 6q21/SE6 probes labeled with SpectrumOrange/SpectrumGreen, $M Y B$ deletion from Cytocell. For each of the probes, the cut-off point value was determined based on the tests carried out on the control group (average $\pm 3 \mathrm{SD}$ ). It was respectively: for the probe for chromosome centromere $12-5 \%$, for the $R B I$ gene probe-10\%, for probes for del(13)(q14.3)(D13S319), del(13)(q34)(LAMP1), del(11)(q22.3)(ATM), del(17)(p13.1) (TP53) it was $8 \%$, and for probes for $\operatorname{del}(6)(\mathrm{q} 21-\mathrm{q} 23)$ it was $3 \%$.

\section{Statistical analyses}

The distribution of the features analyzed was assessed using the Shapiro-Wilk test. The following tests were used: $t$-Student, U Mann-Whitney, $\chi^{2}$ ( $\chi^{2}$ Pearson, $\chi^{2}$ Fisher).

According to the adopted model of research, the odds ratio (OR) and the $95 \%$ confidence interval $(95 \% \mathrm{CI})$ were 
calculated to estimate the association of important features for the clinical course of leukemia with clonal evolution. Survival was calculated using the Kaplan-Meier estimator. The effect of selected features on survival was assessed by the log-rank test and the F Cox test. The logistic regression model (GLZ Multiple Regression, Probit Model, Best subsets) was used to assess the links between the features under analysis and clonal evolution in the CLL process. The calculations were performed using the Statistica 12 software (StatSoft, Inc.).

\section{Results}

Tests with the use of classical cytogenetics and FISH were performed in all the patients at the time of diagnosis and prior to treatment in patients requiring chemotherapy. In order to observe clonal changes (clonal evolution), the tests were repeated. The second test was assumed to be carried out after about 24 months. The time counted in the months between the tests was: median 25.00 (range from 11 to 35 months, average $23.52 \pm 15.00$ ). The shorter time between the tests in some patients was associated with the occurrence of progression. Overall survival (OS) was measured from the diagnosis of CLL and from baseline analysis to the date of the last follow-up visit or death.

\section{Clonal changes detected using classical cytogenetics technique}

In the first test using classical cytogenetics in 33 patients (45.83\%), normal karyotypes were observed. In the remaining patients, detected clonal chromosome aberrations concerned the number and structure of chromosomes; Table 2.

The most commonly appearing chromosome aberration was deletion of the long arm of chromosome 13. It occurred in 14 patients (19.44\%), being the only observed chromosome aberration in 12 of those people (16.67\%). The deletion of chromosome 13 concerned a long-arm fragment with the breakpoints: $\operatorname{del}(13)(\mathrm{q} 14)$ or $\operatorname{del}(13)(\mathrm{q} 14 \mathrm{q} 21)$. Moreover, chromosome 13 was, in two cases, involved in translocations.

Other aberrations that occurred at high incidence included deletion of the long arm of chromosome 11 and trisomy of chromosome 12 .

The deletion of the long arm of chromosome 11 was related to the regions of $\operatorname{del}(11)(q 14)$ or $\operatorname{del}(11)(q 22 q 23)$ and occurred in 5 patients $(6.94 \%)$. The loss of the entire chromosome 11 was observed in one case, and chromosome translocations were also observed in three patients, in which chromosome 11 was involved.

Trisomy of chromosome 12 occurred in 5 patients $(6.94 \%)$.
Changes in chromosome 17 included the loss of the whole chromosome in two cases $(2.78 \%)$.

There were also changes in chromosome 6 in 4 patients (5.55\%). A deletion of a fragment of the long arm of chromosome 6 del(6)(q21) was detected in one patient, and the loss of the whole chromosome 6 was observed in the second patient. In addition, in two cases chromosome 6 was involved in the translocation.

Other interesting changes detected in the karyotype of the subjects were changes related to chromosome 14 .

A complex karyotype was found in the test group in 9 patients $(12.5 \%)$ ) (occurrence of three or more chromosome aberrations).

The second cytogenetic test to analyse karyotypes of the subjects was performed to detect new clonal changes. The emergence of new chromosome aberrations in karyotypes indicates an occurrence of clonal evolution.

During the second test, normal karyotype was observed in 17 patients (23.61\%). Clonal changes appeared in 19 (26.38\%) of the patients who had a normal karyotype in the previous test. However, in 15 people (20.83\%), the second test demonstrated the emergence of new clonal chromosome changes.

A complex karyotype was observed in 15 patients $(20.80 \%)$.

Chromosomal aberrations were related to both the number and structure. The most common was the loss of chromosomes: 13, 14, 16, 17, but there was also trisomy of chromosomes 12, 18 and 21. The structural changes include additions, deletions, and translocations.

An analysis of the chromosome changes found during the first and second cytogenetic test has revealed the existence of clonal evolution in 33 patients (45.8\%). Clonal changes concern both the number and structure of chromosomes. A comparison of the share of individual chromosome aberrations in clonal evolution in CLL patients detected during the two tests is shown in Fig. 1.

\section{Clonal changes detected using the FISH technique}

Owing to the use of a panel of probes to select chromosome areas and centromeric sequences for specific chromosomes, a broad spectrum of chromosome changes have been detected in the genome in the subjects; Table 2.

In the first test, the most frequently observed change was deletion of the long arm of chromosome 13.

In the group tested, in 50 subjects $(69.44 \%)$ a deletion was detected in the region 13q14.3 in the locus D13S319. The deletion occurred as a single change in 25 subjects (34.72\%). In the case of 9 people (12.50\%), the deletion D13S319 was accompanied by a deletion in the $R B 1$ gene (13q14.2), while 13 patients (18\%) had a deletion in D13S319. 
Table 2 Chromosomal aberrations detected in CLL patients using classical cytogenetics and FISH methods

\begin{tabular}{|c|c|c|c|c|}
\hline Item & Karyotype during diagnosis (baseline) & $\begin{array}{l}\text { FISH during } \\
\text { diagnosis (base- } \\
\text { line) }\end{array}$ & FISH, 2nd testing (follow-up) & Karyotype, 2nd testing (follow-up) \\
\hline 1 & $46, X Y[21]$ & $13 q-$ & $13 q-, 6 q-$ & $\begin{array}{l}\text { 46,XY,del(13)(q14q21)[6]/46,sl,del(6)(q24) } \\
\text { [3]/ 46,XY[14] }\end{array}$ \\
\hline 2 & $47, \mathrm{XY},+12[11] / 46, \mathrm{XY}[16]$ & +12 & +12 & $47, \mathrm{XY},+12[14] / 46, \mathrm{XY}[8]$ \\
\hline 3 & $46, \mathrm{XX}[20]$ & $13 q-$ & $13 q-, 17 p-$ & $46, X X, \operatorname{del}(13)(q 14 q 21)[8] / 46, X X[12]$ \\
\hline 4 & $46, X Y, t(9 ; 14)(\mathrm{p} 12 ; \mathrm{q} 32)[8] / 46, \mathrm{XY}[12]$ & $13 q-$ & $13 \mathrm{q}-, 17 \mathrm{p}-$ & $\begin{array}{l}\text { 45,XY,t(9;14)(p12;q32),-13,-16, } \\
\quad+\operatorname{mar}[6] / 46, \mathrm{XY}[15]\end{array}$ \\
\hline 5 & 46,XY, del(13)(q14)[4]/46,XY[24] & $13 \mathrm{q}-, 17 \mathrm{p}-$ & $13 \mathrm{q}-, 17 \mathrm{p}-$ & 45,XY,del(13)(q14),-20[8]/46,XY[12] \\
\hline 6 & $46, X X[30]$ & $13 q-$ & $13 \mathrm{q}-, 17 \mathrm{p}-$ & $46, X X[20]$ \\
\hline 7 & $46, X X[26]$ & $13 q$ biallel & 13q biallel & $46, X X, \operatorname{del}(13)(\mathrm{q} 14)[11] / 46, \mathrm{XX}[9]$ \\
\hline 8 & $46, X Y[19]$ & $13 q-$ & $13 q-$ & $46, X Y[24]$ \\
\hline 9 & 46,XY,del(13)(q14q21)[6]/46,XY[12] & $13 q-, 17 p-$ & $13 q-, 17 p-$ & $\begin{array}{l}\text { 46,XY,del(13)(q14q21)[3]/46,XY,del(13) } \\
\text { (q14q21), del(17)(p12)[4]/46,XY[16] }\end{array}$ \\
\hline 10 & $46, X Y[22]$ & $13 q^{-}, 11 q^{-}$ & $13 q^{-}, 11 q^{-}$ & $\begin{array}{l}\text { 46,XY,del(13)(q14)[7]/46,sl,del(11) } \\
\text { (q22q23)[4]/ 46,XY[8] }\end{array}$ \\
\hline 11 & $46, \mathrm{XX}[27]$ & $\mathrm{N}$ & $\mathrm{N}$ & $46, \mathrm{XX}[23]$ \\
\hline 12 & $46, \mathrm{XX}[23]$ & $13 q^{-}, 11 q^{-}$ & $13 q^{-}, 11 q^{-}$ & $46, X X, \operatorname{del}(13)(q 12 q 14)[5] / 46, X X[26]$ \\
\hline 13 & $46, \mathrm{XX}[24]$ & $13 \mathrm{q}^{-}, 11 \mathrm{q}-$ & $13 q_{-}^{-}, 11 q^{-}$ & 46,XX,del(13)(q12q22)[6]/46,XX[11] \\
\hline 14 & $46, \mathrm{XX}[21]$ & $13 q-,+12$ & $13 \mathrm{q}-,+12$ & $47, \mathrm{XX},+12[4] / 46, \mathrm{XX}[16]$ \\
\hline 15 & $46, X Y[28]$ & $13 q-, 11 q-$ & $13 \mathrm{q}-, 17 \mathrm{p}-$ & $46, \mathrm{XY}[24]$ \\
\hline 16 & $46, X Y[27]$ & $11 q-$ & $11 q-$ & $46, X Y[24]$ \\
\hline 17 & $46, \mathrm{XX}, \operatorname{del}(13)(\mathrm{q} 12)[3] / 46, \mathrm{XX}[20]$ & $13 q-$ & $13 q-$ & 46,XX,del (13)(q12)[6],46,XX[12] \\
\hline 18 & $46, X Y[21]$ & $13 q-$ & $13 q-$ & $46, X Y[20]$ \\
\hline 19 & $46, X Y[26]$ & $17 \mathrm{p}-$ & $6 q-, 17 p-$ & $46, X Y[24]$ \\
\hline 20 & $46, X Y[19]$ & $13 q-$ & $13 q-$ & 46,XY,del(13)(q14)[6]/46,XY[14] \\
\hline 21 & 46,XX,del(13)(q14)[7]/46,XX[13] & $13 q-$ & $13 \mathrm{q}-, 17 \mathrm{p}-$ & $\begin{array}{l}\text { 46,XX,del(13)(q14)[8]/46,sl,del(17)(p12) } \\
\text { [5]/46,XX [9] }\end{array}$ \\
\hline 22 & $46, X Y[22]$ & $13 q-$ & $13 q-$ & $46, X Y[27]$ \\
\hline 23 & $46, \mathrm{XX}, \operatorname{del}(13)(\mathrm{q} 14)[4] / 46, \mathrm{XX}[15]$ & $13 q-$ & $13 q-$ & $46, X X, \operatorname{del}(13)(q 14)[10] / 46, X X[13]$ \\
\hline 24 & $46, X X, \operatorname{del}(13)(q 12 q 34)[3] / 46, X X[15]$ & $13 q^{-}, 11 q^{-}$ & $13 q-$ & 45,X,-X,del(13)(q12q34)[5]/46,XX[16] \\
\hline 25 & 46,XY,del(13)(q14)[8]/46,XY[12] & $13 q-$ & $13 q-$ & $\begin{array}{l}\text { 46,XY,t(6;13)(q15;q14)[2]/46,XY,del(13) } \\
\quad(\mathrm{q} 14)[4] / 46, \mathrm{XY}[12]\end{array}$ \\
\hline 26 & $47, \mathrm{XY},+12[3] / 46, \mathrm{XY}[19]$ & +12 & +12 & $47, X Y,+12[4] / 46, X Y[16]$ \\
\hline 27 & 46,XX,del(13)(q14q21)[5]/46,XX[14] & $13 q-,+12$ & $13 q-,+12$ & $\begin{array}{l}\text { 47,XX, +12,del(13)(q14q21) } \\
{[\mathrm{cp} 6] / 46, \mathrm{XX}[13]}\end{array}$ \\
\hline 28 & $46, X Y[25]$ & $13 q-, 17 p-$ & $13 \mathrm{q}-, 17 \mathrm{p}-$ & $46, X Y[24]$ \\
\hline 29 & $46, \mathrm{XX}[21]$ & $13 q-$ & $13 q-$ & $46, \mathrm{XX}[23]$ \\
\hline 30 & $46, X Y[27]$ & $13 q-$ & 13q biallelic & $46, X Y[25]$ \\
\hline 31 & $46, \mathrm{XX}[22]$ & $13 q-$ & $13 q-$ & $46, X X[20]$ \\
\hline 32 & 46,XX,del(13)(q14)[8]/46,XX[24] & $13 q-$ & $13 q-$ & 46,XX,del(13)(q14)[4]/46,XX[18] \\
\hline 33 & 46,XX,del(13)(q14)[4]/46,XX[16] & $13 q-$ & $13 q-$ & $47, X X, \operatorname{del}(13)(q 14),+\operatorname{mar}[6] / 46, X X[14]$ \\
\hline 34 & $46, X Y[20]$ & $\mathrm{N}$ & $\mathrm{N}$ & $46, X Y[26]$ \\
\hline 35 & $46, X Y[24]$ & $11 \mathrm{q}-$ & $11 \mathrm{q}-$ & $47 \sim 48, \mathrm{XY},+1 \sim 2 \operatorname{mar}[16]$ \\
\hline 36 & $44 \sim 46, \mathrm{XX},-6,-17,+1 \sim 2 \operatorname{mar}[\mathrm{cp} 16]$ & $6 q-$ & $6 q-, 17 p-$ & $44 \sim 46, X X, \operatorname{del}(6)(q 15),-17,+1 \sim 3 \operatorname{mar}[\operatorname{cp} 14]$ \\
\hline 37 & $46, \mathrm{XX}[25]$ & $13 q-$ & 13q biallelic & $46, X X[26]$ \\
\hline 38 & 46,XY,del(14)(q12)[3]/46,XY[22] & $13 \mathrm{q}-, 17 \mathrm{p}-$ & $13 q-, 17 p-$ & $\begin{array}{l}\text { 46,XY,del(14)(q12),der(17)del(17)(p13) } \\
\operatorname{del}(17)(\mathrm{q} 21)[5] / 46, \mathrm{XY}[14]\end{array}$ \\
\hline 39 & $\begin{array}{l}\text { 43,XY,-13, der(15;15)(q10;q10),- } \\
\text { 17[5]/46,XY[10] }\end{array}$ & $13 \mathrm{q}-, 17 \mathrm{p}-$ & $13 q-, 17 p-$ & $\begin{array}{l}\text { 43,XY,-13,der(15;15)(q10;q10),- } \\
\text { 17[6]/46,XY[14] }\end{array}$ \\
\hline
\end{tabular}


Table 2 (continued)

\begin{tabular}{|c|c|c|c|c|}
\hline Item & Karyotype during diagnosis (baseline) & $\begin{array}{l}\text { FISH during } \\
\text { diagnosis (base- } \\
\text { line) }\end{array}$ & FISH, 2nd testing (follow-up) & Karyotype, 2nd testing (follow-up) \\
\hline 40 & $\begin{array}{l}45 \sim 46, X Y, \operatorname{del}(6)(q 21), \operatorname{de}(113)(q 14),- \\
18[\mathrm{cp} 9] / 46, X Y[7]\end{array}$ & $13 q-, 6 q-$ & $13 q-, 6 q-$ & $\begin{array}{l}45 \sim 47, X Y, \operatorname{del}(6)(\mathrm{q} 21), \operatorname{del}(13)(\mathrm{q} 14),-18 \\
\quad+\operatorname{mar}[\mathrm{cp} 5] / \\
46, \mathrm{XY}[16]\end{array}$ \\
\hline 41 & $45, \mathrm{XX}, \mathrm{t}(6 ; 11)(\mathrm{p} 25 ; \mathrm{q} 23),-12[4] / 46, \mathrm{XX}[15]$ & $6 q-$ & $6 \mathrm{q}-$ & $46, \mathrm{XX}, \mathrm{t}(6 ; 11)(\mathrm{p} 25 ; \mathrm{q} 23)[3] / 46, \mathrm{XX}[16]$ \\
\hline 42 & $46, \mathrm{XX}, \operatorname{add}(13)(\mathrm{q} 34)[3] / 46, \mathrm{XX}[20]$ & $\mathrm{N}$ & add 13 & $46, \mathrm{XX}, \operatorname{add}(13)(\mathrm{q} 34)[9] / 46, \mathrm{XX}[12]$ \\
\hline 43 & $47, \mathrm{XX},+21[4] / 46, \mathrm{XX}[27]$ & $\mathrm{N}$ & $\mathrm{N}$ & $\begin{array}{l}\text { 47,XX, +21[5]/46,XX,der(3)t }(3 ; 13) \\
\quad(\mathrm{p} 21 ; \mathrm{q} 14)[5] / 46, \mathrm{XX}[17]\end{array}$ \\
\hline 44 & $46, X Y[30]$ & $\mathrm{N}$ & $\mathrm{N}$ & $46, \mathrm{XY}, \mathrm{t}(18 ; 22)(\mathrm{q} 21 ; \mathrm{q} 11)[6] / 46, \mathrm{XY}[24]$ \\
\hline 45 & $46, X Y[20]$ & $13 q-$ & $13 q-$ & $\begin{array}{l}\text { 46,XY,der(13)del(13)(q14)t(10;13) } \\
\text { (p14;q21)[5]/ 46,XY[25] }\end{array}$ \\
\hline 46 & $46, X Y[12]$ & $13 q-$ & $13 q-$ & $\begin{array}{l}\text { 46,XY,del(13)(q14), add(12)(p13) } \\
\text { [4]/46,XY[12] }\end{array}$ \\
\hline 47 & $\begin{array}{l}46 \sim 48, X X, t(1 ; 12)(q 31 ; q 24), t(6 ; 11) \\
\quad(\mathrm{p} 25 ; \mathrm{q} 23),+12,+18[\mathrm{cp} 8]\end{array}$ & +12 & +12 & $\begin{array}{l}46, \mathrm{XX}, \mathrm{t}(1 ; 12)(\mathrm{q} 31 ; \mathrm{q} 24) \\
\quad+12[6] / 46, \mathrm{sl}, \operatorname{add}(3)(\mathrm{q} 24)[3]\end{array}$ \\
\hline 48 & $46, \mathrm{XX}[17]$ & $11 \mathrm{q}$ & $11 \mathrm{q}-, 17 \mathrm{p}-$ & $49, \mathrm{XX},+8, \operatorname{del}(11)(\mathrm{q} 22),+19,+21[\mathrm{cp} 12]$ \\
\hline 49 & $\begin{array}{l}\text { 45,XX, del(2)(q32q36),-10,-22, } \\
\quad+\text { mar[cp12] }\end{array}$ & $6 q-$ & $6 q-$ & $\begin{array}{l}46, \mathrm{XX}, \operatorname{del}(6)(\mathrm{q} 21)[3] / 46, \mathrm{XX}, \operatorname{del}(2) \\
(\mathrm{q} 32 \mathrm{q} 36),-10,+\operatorname{mar}[8]\end{array}$ \\
\hline 50 & $46, X Y, t(6 ; 13)(q 25 ; q 14)[3] / 46, X Y[14]$ & $13 q-$ & $13 q-$ & $\begin{array}{l}46, \mathrm{XY}, \mathrm{t}(6 ; 13)(\mathrm{q} 25 ; \mathrm{q} 14)[6] / 46, \mathrm{sl}, \operatorname{del}(11) \\
\quad(\mathrm{q} 13),-16,+\operatorname{mar}[9]\end{array}$ \\
\hline 51 & 46,XY,t(2;4)(q21;p14)[3]/46,XY[16] & $13 q-$ & $13 q-$ & $\begin{array}{l}\text { 46,XY,t(2;4)(q21;p14)[4]/46,sl,del (13) } \\
\quad(\mathrm{q} 14 \mathrm{q} 22)[3] / 46, \mathrm{XY}[10]\end{array}$ \\
\hline 52 & 46,XY,del(11)(q14)[5]/46,XY[12] & $11 \mathrm{q}^{-}, 13 \mathrm{q}-$ & $11 \mathrm{q}^{-}, 13 \mathrm{q}-$ & 46,XY,del(11)(q14)[7]/46,XY[13] \\
\hline 53 & $46, X X, \operatorname{del}(13)(q 12 q 22)[6] / 46, X X[12]$ & $13 q-$ & 13q biallelic & 46,XX,del(13)(q12q22)[7]/46,XX[14] \\
\hline 54 & $\begin{array}{l}\text { 46,XY,t(7;13)(q36;q13),del(11)(q14) } \\
{[4] / 46, X Y, \operatorname{del}(11)(\mathrm{q} 14)[2] / 46, X Y[8]}\end{array}$ & $11 q^{-}, 13 q^{-}$ & $11 q^{-}, 13 q^{-}$ & $\begin{array}{l}\text { 46,XY,t(7;13)(q36;q13), del(11)(q14) } \\
\quad[6] / 45, X Y, \operatorname{del}(11)(q 14),-14[3] / 46, X Y[9]\end{array}$ \\
\hline 55 & $\begin{array}{l}45 \sim 46, X,-X, \operatorname{del}(8)(\mathrm{q} 22),-13, \\
\quad+\operatorname{mar}[\mathrm{cp} 6] / 46, \mathrm{XX}[23]\end{array}$ & $13 q-, 17 p-$ & $13 q-, 17 p-$ & $\begin{array}{l}44 \sim 45, X,-X, \operatorname{del}(8)(q 22),-13 \\
{[\mathrm{cp} 7] / 46, X X[18]}\end{array}$ \\
\hline 56 & $\begin{array}{l}44 \sim 45, \mathrm{XY}, \operatorname{del}(8)(\mathrm{p} 23.3 \mathrm{p} 22),-11, \operatorname{del}(13) \\
(\mathrm{q} 14),-15[\mathrm{cp} 11] / 46, \mathrm{XY}[5]\end{array}$ & $13 \mathrm{q}-, 17 \mathrm{p}-,+12$ & $13 q$ biallelic, $17 p,+12$ & $\begin{array}{l}\text { 44 46,XY,del(8)(p23.3p22),-11,del(13) } \\
\quad(\mathrm{q} 14), \operatorname{del}(14)(\mathrm{q} 24),-15,-17 \\
\quad+\operatorname{mar}[\mathrm{cp} 8] / 46, \mathrm{XY}[11]\end{array}$ \\
\hline 57 & 46,XY,del(13)(q14)[3]/46,XY[23] & $13 q-, 11 q-, 17 p-$ & $13 q-$ biallelic, $11 q-, 17 p-$ & 46,XY,del(13)(q14)[7]/46,XY[19] \\
\hline 58 & $46, X Y[24]$ & $13 q-$ & $13 q-$ & $46, X Y[23]$ \\
\hline 59 & $\begin{array}{l}\text { 44,XX, del(1)(q41),del(8)(q24.1), del(9) } \\
\text { (q22), der(10)t(10;11) (p15;q14), del(11) } \\
\text { (q14), add(14)(q23), der(15;15) } \\
\text { (q10;q10), -21[cp6]/ 46,XX[7] }\end{array}$ & $13 q-, 17 p-$ & $13 q-, 17 p-$ & $\begin{array}{l}\text { 46,XX,del(8)(q24.1)[9]/45,XX,del(1) } \\
\text { (q41), del(8)(q24.1),del(9)(q22), der(10) } \\
\text { t(10;11)(p15;q14),del(11)(q14), add(14) } \\
\text { (q23), der(15;15)(q10;q10),-17,-21, } \\
\text { +2mar[cp9]/46,XX[5] }\end{array}$ \\
\hline 60 & $\begin{array}{l}\text { 46,XY,del(11)(q22q23)[7]/46,sl,t(11;14) } \\
\text { (q21;q23)[4]/46,XY[9] }\end{array}$ & $13 q-, 11 q-$ & $13 q-, 11 q-$ & $\begin{array}{l}\text { 46,XY,del(11)(q22q23),t(11;14)(q21;q23) } \\
\text { [6]/46,XY[12] }\end{array}$ \\
\hline 61 & $47, \mathrm{XY},+12[7] / 46, \mathrm{XY}[12]$ & +12 & +12 & $47, \mathrm{XY},+12[5] / 46, \mathrm{XY}[14]$ \\
\hline 62 & $46, \mathrm{XX}[23]$ & +12 & +12 & $46, X X[26]$ \\
\hline 63 & $46, X Y[19]$ & $17 \mathrm{p}-$ & $17 \mathrm{p}-$ & 46,XY,del(17)(p12)[4]/46,XY[16] \\
\hline 64 & $\begin{array}{l}\text { 46,XY,t(3;9)(q13;q34)[7]/45,XY, t(3;9) } \\
(\mathrm{q} 13 ; \mathrm{q} 34), 21[3] / 46, \mathrm{XY}[12]\end{array}$ & $13 q-,+12$ & $13 q-,+12$ & $\begin{array}{l}44 \sim 45, \mathrm{XY}, \mathrm{t}(3 ; 9)(\mathrm{q} 13 ; \mathrm{q} 34),-13,- \\
21[\mathrm{cp} 8] / 46, \mathrm{XY}[16]\end{array}$ \\
\hline 65 & $46, \mathrm{XX}[21]$ & $13 q-, 11 q-$ & $13 q^{-}, 11 q^{-}$ & $46, \mathrm{XX}[25]$ \\
\hline 66 & $46, X X[19]$ & $11 \mathrm{q}-$ & $11 \mathrm{q}-$ & $44 \sim 46, X X,-8,-22,+\operatorname{mar}[\mathrm{cp} 4] / 46, X X[12]$ \\
\hline 67 & $46, X X, t(1 ; 2)(\mathrm{q} 23 ; \mathrm{q} 36)[5] / 46, \mathrm{XX}[14]$ & $13 q-, 11 q-$ & $13 q^{-}, 11 q^{-}$ & $\begin{array}{l}\text { 46,XX,t(1;2)(q23;q36)[3]/46,XX,del(5) } \\
\quad(\mathrm{q} 13 \mathrm{q} 31)[2] / 46, \mathrm{XX}[12]\end{array}$ \\
\hline 68 & $\begin{array}{l}\text { 46,XY,t(2;22)(p21;p11)[5]/45,XY,der(10) } \\
\text { t(10;19)(p14;q13),del(11)(p14),- } \\
\text { 21[4]/46,XY[8] }\end{array}$ & +12 & +12 & $\begin{array}{l}46, \mathrm{XY}, \mathrm{t}(2 ; 22)(\mathrm{p} 21 ; \mathrm{p} 11)[3] / 47, \mathrm{XY} \\
\quad+12[4] / 46, \mathrm{XY}[9]\end{array}$ \\
\hline
\end{tabular}


Table 2 (continued)

\begin{tabular}{|c|c|c|c|c|}
\hline Item & Karyotype during diagnosis (baseline) & $\begin{array}{l}\text { FISH during } \\
\text { diagnosis (base- } \\
\text { line) }\end{array}$ & FISH, 2nd testing (follow-up) & Karyotype, 2nd testing (follow-up) \\
\hline 69 & $\begin{array}{l}\text { 46,XY,t(9;10)(q12;q25),- } \\
\text { 13[4]/46,XY,del(12)(q23)[3]/46,XY[23] }\end{array}$ & $13 q-, 11 q-$ & $13 q-, 11 q-$ & $\begin{array}{l}\text { 46,XY,t(9;10)(q12;q25)[7]/46,XY,del(12) } \\
(\mathrm{q} 23)[5] / 46, X Y, t(2 ; 3)(\mathrm{p} 25 ; \mathrm{p} 14) \\
{[3] / 45, \mathrm{XY},-13[3] / 46, \mathrm{XY}[14]}\end{array}$ \\
\hline 70 & $\begin{array}{l}45, \mathrm{XX}, \operatorname{del}(2)(\mathrm{q} 22),-14[6] / 46, \mathrm{XX}, \operatorname{del}(9) \\
(\mathrm{q} 21)[3] / 46, \mathrm{XX}[20]\end{array}$ & +12 & +12 & $45, \mathrm{XX}, \operatorname{del}(2)(\mathrm{q} 22),-14[3] / 46, \mathrm{XX}[22]$ \\
\hline 71 & $\begin{array}{l}46, \mathrm{XX}, \mathrm{t}(11 ; 12)(\mathrm{q} 14 ; \mathrm{p} 13) \\
{[5] / 46, \mathrm{XX}, \mathrm{t}(1 ; 16)(\mathrm{p} 33 ; \mathrm{p} 13)[4] / 49, \mathrm{XX},} \\
\quad+12,+18,21[3] / 46, \mathrm{XX}[15]\end{array}$ & +12 & +12 & $\begin{array}{l}46, \mathrm{XX}, \mathrm{t}(11 ; 12)(\mathrm{q} 14 ; \mathrm{p} 13) \\
\quad+18[5] / 48, \mathrm{XX}, \mathrm{t}(9 ; 16)(\mathrm{p} 10 ; \mathrm{q} 10),+12 \\
\quad+18[4] / 46, \mathrm{XX}[20]\end{array}$ \\
\hline 72 & $46, X X[22]$ & $13 q-, 11 q-$ & $13 q-, 11 q-$ & 46,XX,t(4;13)(q28,q34)[5]/46,XX[23] \\
\hline
\end{tabular}

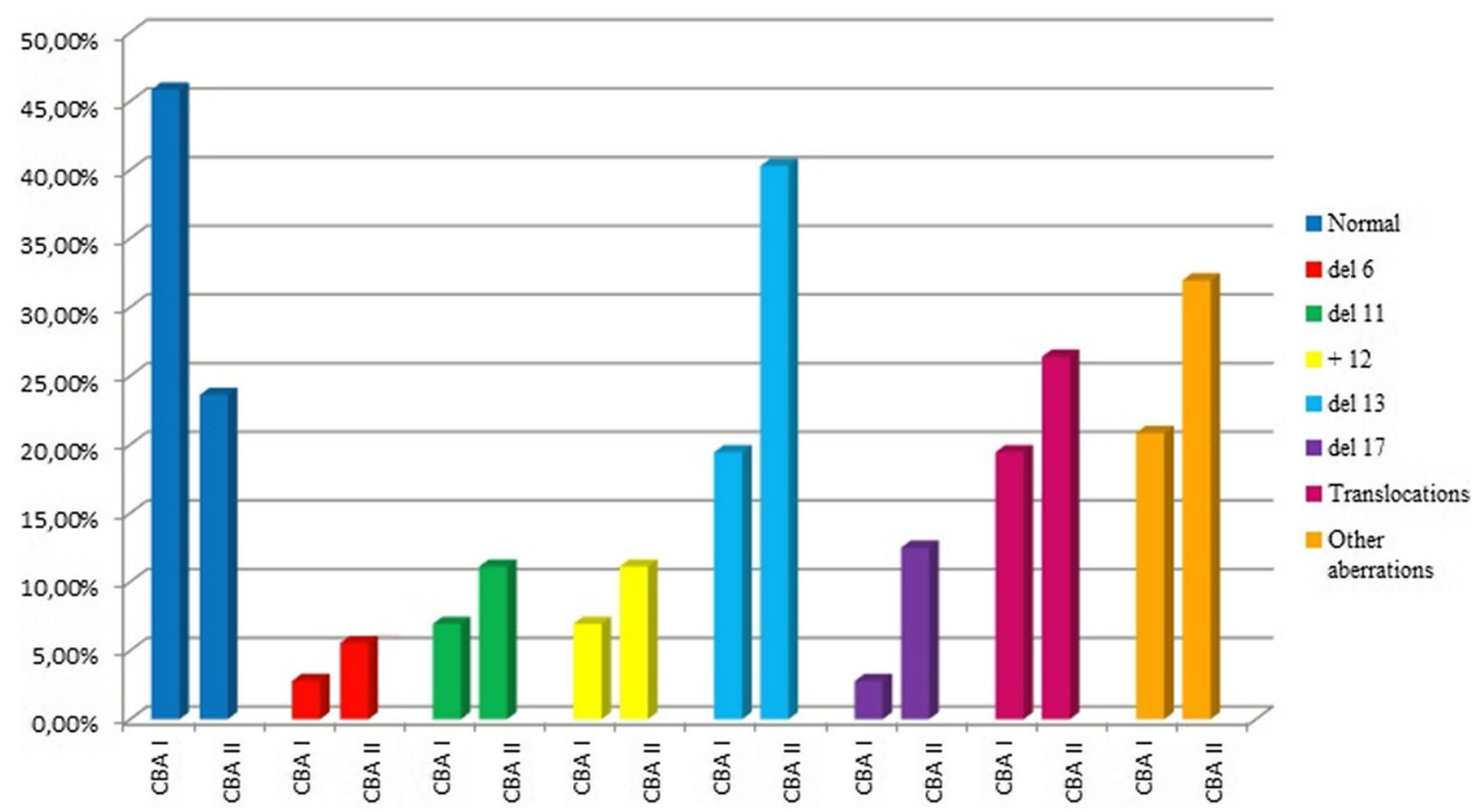

Fig. 1 Comparison of the shares of selected chromosomal aberrations in CLL patients, detected during the first and second tests of karyotype (Color figure online)

The second most frequently observed aberration was a deletion of the long arm of chromosome 11. A deletion of the long arm of chromosome 11 containing ATM (11q22.3) was observed in 17 patients $(23.61 \%)$.

Another change that was detected with high frequency was the trisomy of chromosome 12 . The presence of an additional copy of chromosome 12 was observed in 12 patients (16.67\%). In 8 patients $(11.11 \%)$, it was the only chromosomal aberration detected.

In the first test, a deletion in the short arm of chromosome 17 was detected in 11 patients $(15.27 \%)$. The most frequently occurring aberration accompanying a deletion of
$17 p$ was a deletion of $13 q$, and these changes were detected in 7 patients $(9.72 \%)$.

Another aberration was a deletion of the long arm of chromosome 6. Del(6)(q21) was observed in 4 patients (5.56\%). In the case of five patients (6.94\%) no genomic changes were observed at the level of resolution of the method.

In the second test (during follow-up observation), no chromosomal changes were observed in four patients $(5.55 \%)$, and a deletion of a fragment $13 \mathrm{q}$ was detected in 50 patients (69.44\%). In 22 patients (30.55\%), a del13q occurred as a single chromosomal aberration. In the whole group, in seven patients $(9.72 \%)$ the occurrence of monoand bi-allelic deletions were observed at the locus D13S319. 


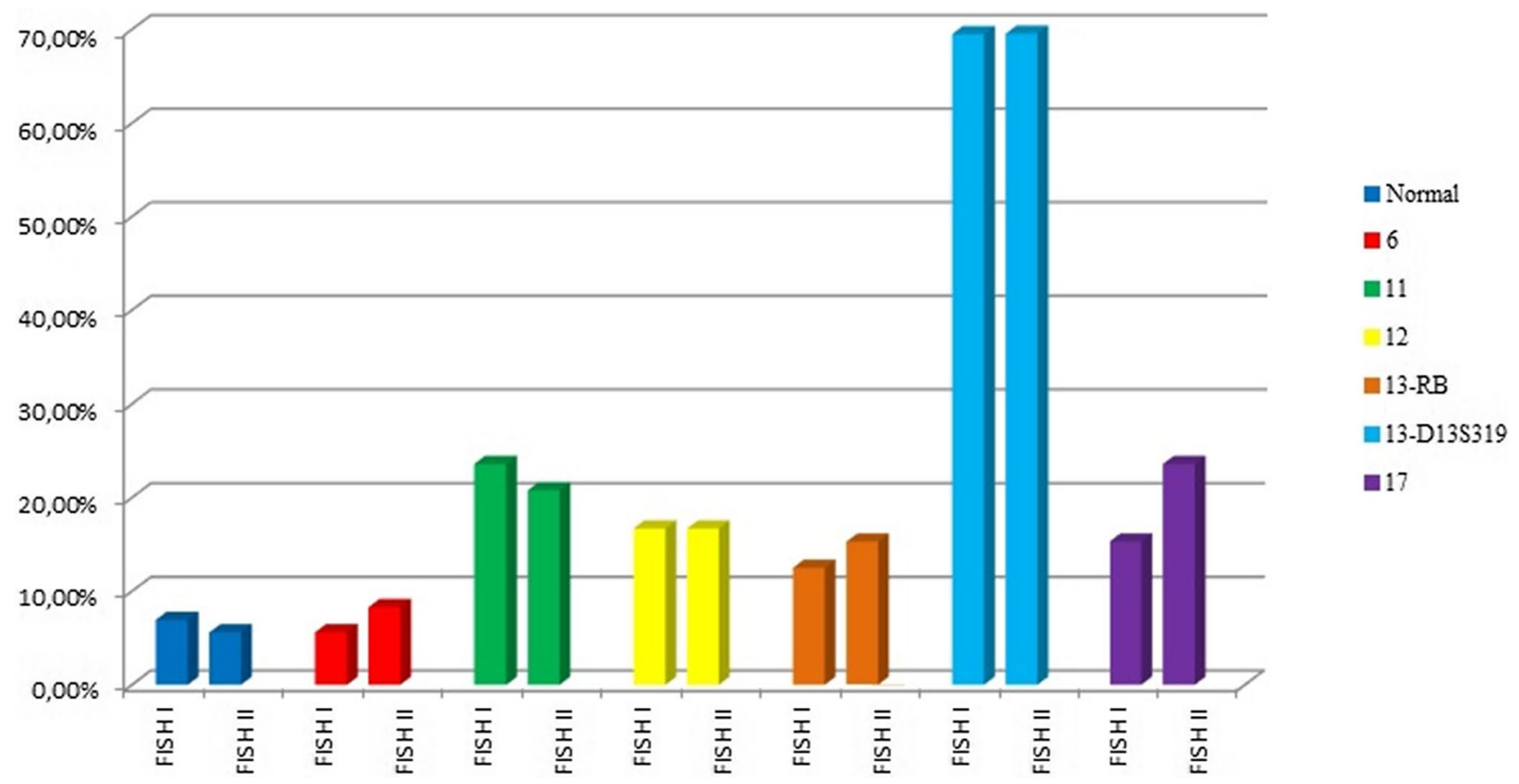

Fig. 2 Comparison of chromosomal changes detected with the FISH technique during the 1st and 2nd tests in CLL patients (Color figure online)

The second most common aberration detected during the second test was a deletion of $17 \mathrm{p} 13$ which was found in 17 patients (23.61\%) and a deletion of 11q was observed in 15 patients $(20.83 \%)$. A deletion of $11 \mathrm{q}$ as a single aberration was observed in 3 patients.

In the next test, the trisomy of chromosome 12 as the only observed aberration was detected in 12 patients $(16.67 \%)$.

The loss of a fragment of the long arm of chromosome 6 was found in 6 patients $(8.33 \%)$.

Owing to the use of the FISH technique, clonal evolution was detected in 14 patients (19.44\%). Clonal changes were associated with the emergence of a deletion of chromosome $17 p$ in 6 patients, deletion of chromosome $6 q$ in two patients, and changes in within chromosome 13: deletion of the $R B I$ gene in two patients and a bi-allelic deletion of the region 13q14.2 (D13S319) in 7 patients.

In addition, 6 patients with clonal evolution (deletion of TP53) were in a high-risk group according to the FISH hierarchical risk category according to the Döhner's classification [10]. The comparison of chromosomal changes detected with the FISH technique during the 1st and 2nd tests is presented in Fig. 2.

Clonal evolution (CE) was detected in 33 (45.8\%) of patients after the application of classic cytogenetics techniques, and in 14 patients (19.4\%) after application of the FISH technique.

A comparison of the number (changes) of particular chromosome aberrations detected by both techniques is shown in Table 3.
Table 3 Cytogenetic abnormalities detected by CBA and FISH

\begin{tabular}{lcccc}
\hline Genetic markers & CBA I (N) & CBA II (N) & FISH I (N) & FISH II (N) \\
\hline $\begin{array}{l}\text { del 17p and del } \\
\text { TP53 mono- }\end{array}$ & 2 & 9 & 11 & 17 \\
$\quad$ & & & \\
somy 17 & 5 & 8 & 17 & 15 \\
del 11q & 5 & 8 & 12 & 12 \\
Trisomy 12 & 14 & 29 & 50 & 50 \\
del 13q & 2 & 4 & 4 & 6 \\
del 6q & 9 & 15 & - & - \\
$\begin{array}{l}\text { Complex karyo- } \\
\text { type }\end{array}$ & & & & \\
\hline
\end{tabular}

$N$ number of cases

\section{Clonal evolution and mutation status of the genes IGVH and NOTCH1}

The tests of mutation status of the $I G V H$ genes performed using the sequencing method allowed us to detect changes in the sequence of examined genes in 12 patients (16.7\%), and in $60(83.3 \%)$ patients, no mutations were detected.

The performed cytogenetic tests allowed for the identification of occurrence of chromosomal aberrations in 11 patients with the mutation of $I G V H$. Only in the case of one patient with a mutation, no changes were detected after applying both research techniques. Analysis of the results obtained clearly indicates an increase in the number of chromosomal aberrations in patients without mutations in the $I G V H$ genes. 
Studies on the mutational status of $I G V H$ genes did not show a statistically significant correlation between the presence of mutations and Rai stage of the disease, age, and sex of patients and the analyzed clinical parameters.

Sequencing of a fragment of the NOTCHI gene, allowed us to detect changes in the sequence of a fragment of exon 34 in 8 patients. All the subjects in whom the mutation was detected had the same change, i.e., a deletion of two nucleotides: cytosine and thymine at position 7544_7545 (75447545delCT, P2515fs).

Mutation in the NOTCHI gene was statistically more frequent in men $(p<0.05)$, but was not statistically significantly related to the age of patients with CLL. Having performed cytogenetic tests, it was found that the trisomy of chromosome 12 occurred statistically significantly more often in patients with a mutation in NOTCH1 (OR $=7.00 ; p<0.05)$. Moreover, it was observed that a deletion of a fragment of the long arm of chromosome $13 \mathrm{del}(13 \mathrm{q})$ is more common in patients with no mutations in the NOTCHI gene $(\mathrm{OR}=2.56)$.

The mutation of NOTCH1 was not related in a statistically significant way to clonal evolution evaluated in particular techniques.

\section{Correlations between clonal evolution and cytogenetics and clinical parameters}

The expression of the CD38 surface antigen in the group of subjects with CLL was categorized in accordance with the applicable clinical standard, which allowed dividing the subjects into two groups, i.e., those with low expression $(<30 \%)$ and high expression ( $>30 \%)$. In the test group, patients with high CD38 expression constituted $36.11 \%$ while patients with no CD38 expression-63.89\%. The tests using cytogenetic techniques confirmed statistically significantly more frequent occurrence of a deletion of 13q14 (D13S319) in patients with low expression of the CD38 antigen $(p<0.001)$. It was also found that high expression of CD38 is a risk factor for the occurrence of chromosome 12 trisomy $(\mathrm{OR}=3.02)$. The other clonal changes assessed by these methods were not statistically significantly related to CD38 expression. The categorized values of CD38 expression were not statistically significantly related to clonal evolution evaluated by specific techniques.

In the test group, ZAP-70 positive patients constituted $47.22 \%$ while patients with no ZAP-70 expression-52.78\%.

FISH tests have shown that the deletion of chromosome $11 \operatorname{del}(11 \mathrm{q})$ is statistically more frequent in patients with high-expression of ZAP-70 $(p<0.05)$. It was also found that high ZAP-70 values are a risk factor for the occurrence of a deletion of $6 \mathrm{q} 22(\mathrm{OR}=2.40)$. The categorized values of ZAP-70 expression were not statistically significantly related to clonal evolution evaluated by specific techniques.
Within the group, 57 patients $(79.2 \%)$ were provided treatment due to the progressive nature of the disease. This group comprised 25 women and 32 men and the average age was 63.60 years.

The time to treatment was 12.54 months on average (0.00- 110 months).

Further observation allowed for the conclusion in 15 patients $(26.32 \%)$ complete remission, and 32 patients (56.14\%) had partial remission. Progression occurred in 10 patients (17.54\%).

Testing of clonal changes with cytogenetic methods allowed us to observe statistically significantly more frequent occurrence of a deletion of $13 \mathrm{q}(p<0.01)$ in patients with complete remission. The other clones assessed using this technique were not significantly linked to the total remission in the subjects.

Having conducted statistical analyses, it was found that the complete remission was not related statistically with clonal evolution in the group of subjects covered by treatment.

There was no significant link between progression and subjects' sex, but progression occurred more statistically significantly in older patients $(p<0.01)$. The analysis of clonal changes in the group of CLL patients treated with cytostatics has demonstrated statistically significantly more frequent occurrence of progression, in the case of the emergence of clones with a deletion of $17 \mathrm{p}$ (deletion of TP53) $(p<0.05)$. The results obtained with the FISH technique have unambiguously indicated the deletion of $17 \mathrm{p}$ as a progression risk factor $(\mathrm{OR}=4.17)$. The other clones assessed by all the techniques were not significantly linked to the progression of the disease. The measured OR values for the deletion of $11 \mathrm{q}(\mathrm{OR}=4.92)$ and $17 \mathrm{p}(\mathrm{OR}=4.00)$ in classical cytogenetic tests indicate that these aberrations constitute a progression risk factor.

The statistical analyses carried out point to clonal evolution as a risk factor for the occurrence of progression $(\mathrm{OR}=2.22)$.

The average overall survival of the patients covered by the observation was 67.40 months (median 55.50 months, [11-240 months]). A total of 34 patients died during the observation. Statistically significant effects of age, sex and stage of disease according to the classification by Rai et al. have been demonstrated.

Comparing the relationships between individual chromosomal aberrations detected using methods of classical cytogenetics and FISH, and the overall survival, a statistically significant relationship was found at the occurrence of $17 p$ (del TP53) $(p<0.0018)$ (Fig. 3), chromosomal translocations and the appearance of complex karyotypes during observation of clonal evolution $(p<0.0423)$. 
Fig. 3 Overall survival in patients with and without $17 \mathrm{p} 13$ deletion

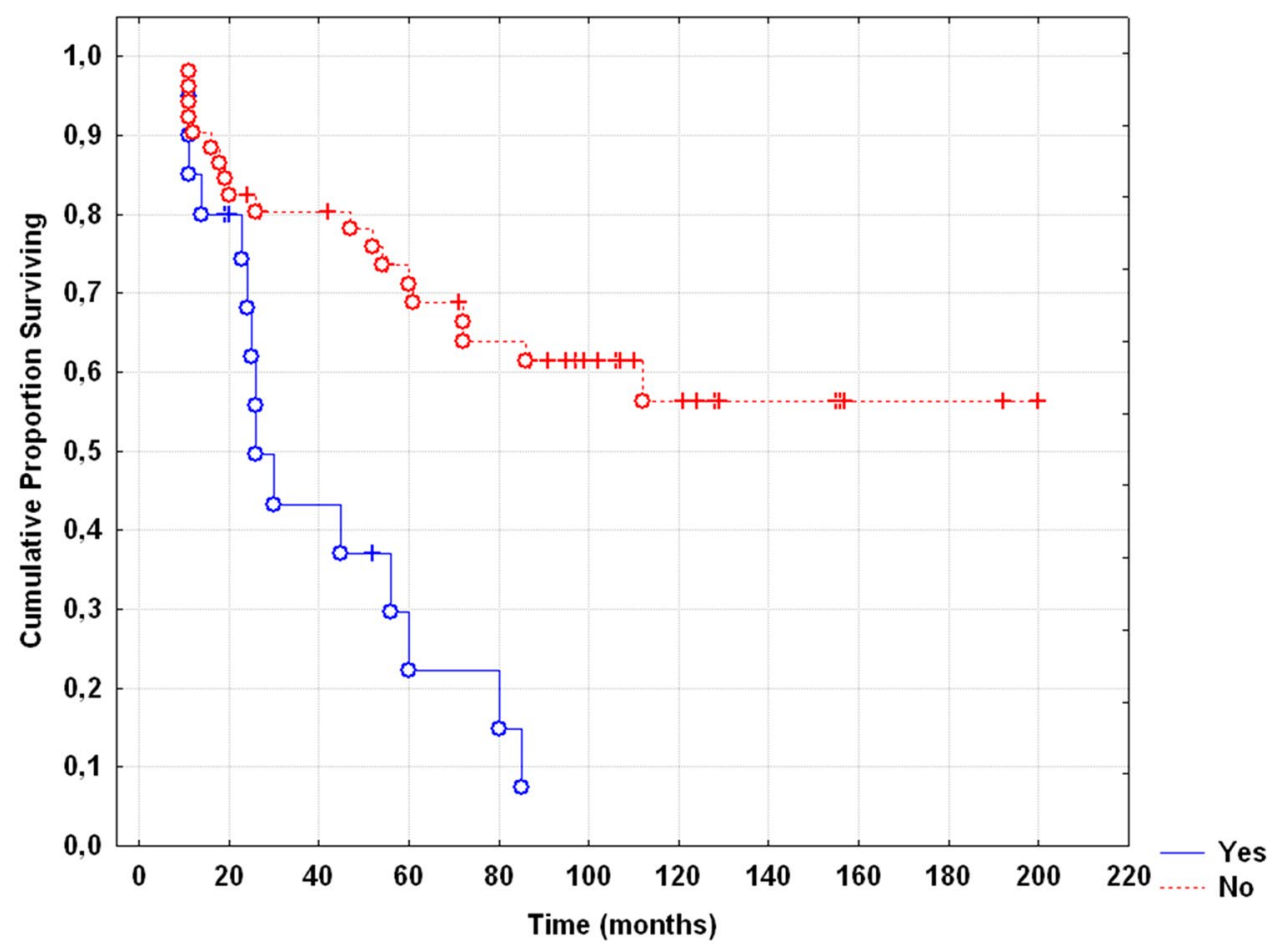

Clonal evolution assessed by methods of classical cytogenetics and the FISH method did not significantly affect the survival of patients with CLL.

\section{Discussion}

Genomic research on cancer cells that have been conducted in recent years have allowed noticing high heterogeneity and the presence of clonal populations $[15,16]$. It was considered that probably clonal evolution in tumors takes place as a result of competition and interaction between genetically diversified cell clones [17]. The incidence, diversity, and evolutionary dynamics of clonal changes in CLL may contribute to the rate of progression of the disease and response to treatment [18]. In the observation of the process of clonal evolution in CLL essential is the slow growth of leukemia cells compared to other malignant tumors because it may take many months or years before a new cell clone fully replaces the previous clones [18, 19].

The acquisition of chromosome anomalies during the course of the disease (clonal evolution - CE) was a rare phenomenon in CLL when using classical cytogenetic methods [20]. The detection of these changes has increased dramatically in recent years by the introduction of stimulating agents, such as DSP30/IL2, in cell cultures. [21]. The phenomenon of clonal evolution was more frequently observed with the use of a more sensitive method, namely the FISH technique $[20,22,23]$.
There are few reports in the literature on the subject, describing first attempts to compare these two methods in assessing clonal evolution [12, 24]. In our own studies, CE was more often detected by classical cytogenetics methods than by FISH ( $45.8 \%$ vs. $19.4 \%)$. The results obtained do not differ from the results obtained by other authors. Fegan et al. [25] observed CE with classical cytogenetics methods in $43 \%$ of patients with CLL [24]. Haferlach et al. observed clonal evolution detected with classical cytogenetic techniques in $30 \%$ of patients, and with the FISH technique in $11.16 \%$ [26]. On the other hand, Wawrzyniak et al. detected $\mathrm{CE}$ in $39.5 \%$ of patients with classical techniques and in $26.3 \%$ of patients with the FISH technique [24].

Our research has shown that the majority of chromosome disorders acquired during $\mathrm{CE}$, detected by classical cytogenetic tests, are structural aberrations including the following deletions: 13q, 17p, 11q, 6q, and translocations involving the chromosomes: $2,3,4,6,8,9,10,11,13,14$, and 22. Additional copies of chromosomes 12, 18, and 19 and marker chromosomes were also observed. On the other hand, the most frequently observed CE-related clonal changes detected using FISH are mono- and bi-allelic deletion of $13 q 14$, deletion of $17 p 13$, and deletion of $6 q$.

In our research, a comparison of both methods in the detection of so-called high-risk aberrations, i.e., deletions of TP53 or ATM detected with FISH and deletions of $11 \mathrm{q} 22$ or abnormalities of $17 \mathrm{p}$ detected using methods of classical cytogenetics have shown that FISH is more accurate and reliable in detecting high-risk changes. 
This aspect of testing is clinically significant due to the time and type of treatment being introduced.

The recent literature on the subject point to the value of classical cytogenetic studies in accurate determining the prognosis for CLL patients [27-30].

Rigolin et al. have demonstrated that classical cytogenetic tests are more effective than FISH in detecting complex karyotypes. The presence of complex karyotypes is of a prognostic nature and affects TFT, which was confirmed in clinical observations [28].

Our tests showed in 7 patients $(9.7 \%)$ the incidence of a complex karyotype during clonal evolution. The group of these patients was characterized by the early administration of treatment (shorter TFT), which is consistent with the observations of Rigolin et al. [28].

It should be noted that in the course of the observation in the group of CLL patients there was no evidence of the acquisition of trisomy of chromosome 12 , which correlates with reports in the literature [29, 30].

In our research no relationship was found between clonal evolution and the mutation status in the $I G V H$ genes.

Clonal evolution was observed in 8 patients with mutations in the $I G V H$ genes.

Multicenter research on clonal evolution and its relationship with the mutation status in the $I G V H$ genes provide interesting results [26, 31]. The studies by Parikh et al. and their extensive literature review evaluating the prognostic relevance of mutation status of the $I G V H$ genes and the studies using FISH to perform stratification of patients with newly diagnosed and/or previously untreated CLL indicate their reliable and coherent prognostic values [32]. Delgado et al. developed a prognostic system that depended on biomarkers for CLL patients based on the two most important prognostic parameters for this leukemia, i.e., the mutation status of $I G V H$ genes and chromosomal aberration tests using the FISH technique. The researchers distinguished three clinical risk groups. The model developed by them can be extremely useful in clinical practice and used to stratify patients in clinical tests [33].

A correlation between the occurrence of mutations in the NOTCH1 gene and the presence of trisomy of chromosome $12(p<0.05)$ was observed in the studies.

Many research teams dealing with similar topics found the occurrence of mutations in the NOTCHI gene in a small percentage of patients with CLL and these values ranged between 4.6 and 12\% [34-36]. Balatti et al. [37] observed an increase in the percentage of patients with a mutation in the NOTCHl gene up to $42 \%$ in cases where the mutation was accompanied by trisomy of chromosome 12 . There was also a high frequency of this mutation in patients where trisomy of chromosome 12 was accompanied by high expression of
ZAP-70 and there was no mutation in the $I G V H$ genes [5, 38].

The occurrence of NOTCH1 mutations in CLL patients is considered an unfavourable prognostic factor. The mutation of NOTCH1 is very often accompanied by high expression of the CD38 antigen and the ZAP-70 kinase. It was also observed that in most patients with mutations in NOTCHI there were no somatic mutations in $I G V H$, which was associated with adverse prognosis. In addition, attention was drawn to the lack of coexistence of mutations in NOTCHI in patients' cells and the loss of the TP53 gene or changes in its sequence, the presence of which are an indicator of poor prognosis and shortened survival time [34, 39]. Our own studies did not show statistically significant correlations between clinical/laboratory parameters and the $\mathrm{NOTCHI}$ mutation status in the group of CLL patients. Our studies did not show the presence of somatic mutations in genes for the immunoglobulin heavy chain variable region in patients with a mutation of NOTCH1. Multicentre observations point to significant correlations between the occurrence of mutations in the NOTCHI gene and cytogenetic aberrations occurring in the course of CLL, especially trisomy of chromosome 12. Co-occurrence of this mutation and trisomy 12 in CLL leukemic cells is associated with an unfavorable prognosis and shortening of overall survival [35-41].

The existence of correlation between the incidence of mutations in the NOTCHI gene and the presence of trisomy of chromosome 12 was confirmed. This correlation has been confirmed in both research techniques. No relationship between mutations in the NOTCHI gene and clonal evolution has been found in our research.

Particular attention should be paid to the results of statistical analyses that indicated clonal evolution as a risk factor for the progression in CLL patients. The literature confirm the increased risk of progression in patients with clonal evolution [5, 42]. In our study, the analysis of clonal changes in the group of patients covered by treatment has demonstrated statistically significantly the incidence of progression, when the appearance of clones with a deletion of $17 p$ was observed (deletion of TP53) $(p<0.05)$. The emergence of deletions of $11 \mathrm{q}$ and $17 \mathrm{p}$ during clonal evolution was indicated as risk factors for progression. Further analyses indicated the phenomenon of clonal evolution as a risk factor for progression.

In our study, clonal evolution has not significantly affected the survival of the CLL patients during the periods under analysis.

The statistically significant lower OS rates were found in patients with a deletion of $17 \mathrm{p}(p<0.0018)$ and chromosomal translocations $(p<0.0423)$ occurring alone or in complex karyotypes. Similar observations have been published by Wawrzyniak et al. who have not found significant differences in the length of OS in patients with or without 
clonal evolution [24]. Also Haferlach et al. made similar observations [26]. Lopez et al. in their study observed shorter TFS in patients who had acquired new chromosome abnormalities, but clonal evolution did not have a significant impact on their overall survival time [43].

However, studies conducted by Janssens et al. and Shanafelt et al. showed a relationship between clonal evolution and shortened survival time [29, 44].

Failure to note the effect of clonal evolution detected with tests of classical cytogenetics and FISH on the survival of CLL patients may result from the presence of other newly acquired aberrations (gene mutations) impossible to detect with these techniques. It is possible that these are mutations of such genes as TP53, ATM, BIRC3, SFB1, MYD88, POT1, CHD2, XPO1, FBXW7, DDX3X, EGR2, NFKBIE, or SETD2 [45].

The results obtained showed that conventional karyotyping should be included in the observation of clonal evolution in addition to the FISH technique. These observations confirm the suggestions of many researchers that the assessment of risk of the clinical course of the disease and monitoring the treatment of patients with CLL indicate the need to include conventional karyotyping for screening these patients, similarly to those routinely performed in other hematological disorders [21, 28].

Acknowledgements The authors would like to thank M. Luterek, I. Pilecka, MSc, and M. Wojcierowska-Litwin, MSc for help in performed chromosome banding and genes sequencing.

Author contributions DK, SP-M, and SZ designed the study, and EWS collected patients' data. DK wrote the manuscript, and AF revised and finally approved of the version to be submitted.

Data availability Please contact with authors for any data.

\section{Compliance with ethical standards}

Conflict of interest The authors declare no conflict of interest.

Ethic approval Informed consent in accordance with the Declaration of Helsinki of 1975, revised in 2008 and after approval from the Medical University Ethics Committee (app. no.: KE-0254/153/2010) was obtained from all patients.

Informed consent All patients provided informed written consent for all procedures in the study.

Open Access This article is distributed under the terms of the Creative Commons Attribution 4.0 International License (http://creativeco mmons.org/licenses/by/4.0/), which permits unrestricted use, distribution, and reproduction in any medium, provided you give appropriate credit to the original author(s) and the source, provide a link to the Creative Commons license, and indicate if changes were made.

\section{References}

1. Dighiero G. CLL biology and prognosis. Hematol Am Soc Hematol Educ Program. 2005;2005:278-84. https://doi.org/10.1182/ asheducation-2005.1.278.

2. Rai K, Jain P. Chronic lymphocytic leukemia (CLL) - then and now. AM J Haematol. 2016;91(3):330-40. https://doi.org/10.1002/ ajh.24282.

3. Hallek M, Cheson BD, Catovsky D, et al. iwCLL guidelines for diagnosis, indications for treatment, response assessment, and supportive management of CLL. Blood. 2018;131(25):2745-60.

4. Eichhorst B, Hallek M. Prognostication of chronic lymphocytic leukemia in the era of new agents. Hematol Am Soc Hematol Educ Program. 2016;2016:149-55.

5. Del Giudice I, Rossi D, Chiaretti S, Marinelli M, Tavolaro S, Gabrielli S, Laurenti L, Marasca R, Rasi S, Fangazio M, Guarini A, Gaidano G, Foa R. NOTCH1 mutations in +12 chronic lymphocytic leukemia (CLL) confer an unfavorable prognosis, induce a distinctive transcriptional profiling and refine the intermediate prognosis of +12 CLL. Haematologica. 2012;97:437-41. https:// doi.org/10.3324/haematol.2011.060129.

6. Finn WG, Kay NE, Kroft SH, Church S, Peterson LC. Secondary abnormalities of chromosome $6 \mathrm{q}$ in B-cell chronic lymphocytic leukemia: a sequential study of karyotypic instability in 51 patients. Am J Hematol. 1998;59:223-9.

7. Juliusson G, Oscier D, Gahrton G, for the International Working Party On Chromosomes In CLL (IWCCLL). Cytogenetic findings and survival in B-cell chronic lymphocytic Leukemia. Second IWCCLL compilation of date on 662 patients. Leukemia Lymph. 1991;5:21-5.

8. Bercova A, Zemanova Z, Trneny M, Schwartz J, Karban J, Cmunt E, Pavlistova L, Brezinova J, Michalova K. Clonal evolution In chronic lymphocyytic leukemia studied by interphase fluorescence in situ hybridization. Neoplasma. 2009;56(5):455-8.

9. Haferlach C, Dicker F, Schnittger S, Kern W, Haferlach T. Comprehensive genetic characterization of CLL a study on 506 cases analyzed with chromosome banding analysis, interphase FISH, IGVH status and immunophenotyping. Leukemia. 2007;21:244251. https://doi.org/10.1038/sj.leu.2404935.

10. Döhner H, Stilgenbauer S, Benner A, Leupolt E, Krober A, Bullinger L, Dohner K, Bentz M, Lichter P. Genomic aberrations and survival in chronic lymphocytic leukemia. N Eng J Med. 2000;343:1910-6. https://doi.org/10.1056/nejm20001228343 2602.

11. Hallek M, Cheson BD, Catovsky D, Caligaris-Cappio F, Dighiero G, Döhner H, Hillmen P, Keating M, Montserrat E, Chiorazzi N, Stilgenbauer S, Rai KR, Byrd JC, Eichhorst B, O'Brien S, Robak T, Seymour JF, Kipps TJ. Guidelines for diagnosis, indications for treatment, response assessment and supportive management of chronic lymphocytic leukemia. Blood. 2018. https://doi. org/10.1182/blood-2017-09-806398.

12. López C, Delgado J, Costa D, Villamor N, Navarro A, Cazorla M, Gómez C, Arias A, Muñoz C, Cabezas S, Baumann T, Rozman M, Aymerich M, Colomer D, Pereira A, Cobo F, López-Guillermo A, Campo E, Carrió A. Clonal evolution in chronic lymphocytic leukemia: analysis of correlations with IGHV mutational status, NOTCH1 mutations and clinical significance. Genes Chromosomes Cancer. 2013;52(10):920-7. https://doi.org/10.1002/ gcc. 22087.

13. Koczkodaj D, Popek S, Zmorzyński S, Wąsik-Szczepanek E, Filip AA. Detection of chromosomal changes in chronic lymphocytic leukemia using classical cytogenetic methods and FISH: application of rich mitogen mixtures for lymphocyte cultures. J Invest Med. 2016;64:894-8. https://doi.org/10.1136/jim-2015-000050. 
14. McGowan-Jordan J, Simons A, Schmid M, eds. ISCN 2016: An International System for Human Cytogenomic Nomenclature. Basel (Switzerland) Karger 2016 ISBN 978-3-318-05857-4.

15. Ding L, Ley TJ, Larson DE, Miller CA, Koboldt DC, Welch JS, Ritchey JK, Young MA, Lamprecht T, McLellan MD, McMichael JF, Wallis JW, Lu C, Shen D, Harris CC, Dooling DJ, Fulton RS, Fulton LL, Chen K, Schmidt H, Kalicki-Veizer J, Magrini VJ, Cook L, McGrath SD, Vickery TL, Wendl MC, Heath S, Watson MA, Link DC, Tomasson MH, Shannon WD, Payton JE, Kulkarni S, Westervelt P, Walter MJ, Graubert TA, Mardis ER, Wilson RK, DiPersio JF. Clonal evolution in relapsed acute myeloid leukaemia revealed by whole-genome sequencing. Nature. 2012;481(7382):506-10. https://doi.org/10.1038/nature10738.

16. Gerlinger M, Rowan AJ, Horswell S, Math M, Larkin J, Endesfelder D, Gronroos E, Martinez P, Matthews N, Stewart A, Tarpey P, Varela I, Phillimore B, Begum S, McDonald NQ, Butler A, Jones D, Raine K, Latimer C, Santos CR, Nohadani M, Eklund AC, Spencer-Dene B, Clark G, Pickering L, Stamp G, Gore M, Szallasi Z, Downward J, Futreal PA, Swanton C. Intratumor heterogeneity and branched evolution revealed by multiregion sequencing. N Engl J Med. 2012;366(10):883-92. https://doi.org/10.1056/ nejmoa1113205.

17. Snuderl M, Fazlollahi L, Le LP, Nitta M, Zhelyazkova BH, Davidson CJ, Akhavanfard S, Cahill DP, Aldape KD, Betensky RA, Louis DN, Iafrate AJ. Mosaic amplification of multiple receptor tyrosine kinase genes in glioblastoma. Cancer Cell. 2011;20(6):810-7. https://doi.org/10.1016/j.ccr.2011.11.005.

18. Schuh A, Becq J, Humphray S, Alexa A, Burns A, Clifford R, Feller SM, Grocock R, Henderson S, Khrebtukova I, Kingsbury Z, Luo S, McBride D, Murray L, Menju T, Timbs A, Ross M, Taylor J, Bentley D. Monitoring chronic lymphocytic leukemia progression by whole genome sequencing reveals heterogeneous clonal evolution patterns. Blood. 2012;120:4191-6. https://doi. org/10.1182/blood-2012-05-433540.

19. Wu CJ. CLL clonal heterogeneity: an ecology of competing subpopulations. Blood. 2012;120(20):4117-8. https://doi. org/10.1182/blood-2012-09-452805.

20. Cotter M, Auer RL. Genetic alterations associated with chronic lymphocytic leukemia. Cytogenet Genome Res. 2007;118:310-9. https://doi.org/10.1159/000108315.

21. Dubuc A, Davids M, Pulluqi M, Hoang K, Hernandez-Sanchez J, Schlich C, Hernandez-Rivas M, Brown J, Dal Cin P. FISHing in the dark: How the combination of FISH and conventional karyotyping improves the diagnostic yield in $\mathrm{CpG}$-stimulated chronic lymphocytic leukemia. Am J Hematol. 2016;91(10):978-83. https ://doi.org/10.1002/ajh.24452.

22. Bercova A, Zemanova Z, Trneny M, Schwartz J, Karban J, Cmunt E, Pavlistova L, Brezinova J, Michalova K. Clonal evolution In chronic lymphocyytic leukemia studied by interphase fluorescence in situ hybridization. Neoplasma. 2009;56:455-8.

23. Loscertales J, Arranz E, Sanz MA, Blas C, Gil-Fernández JJ, Burgaleta C, Alegre A. Clonal evolution in patients with chronic lymphocytic leukemia. Leuk Lymphoma. 2010;51:1142-3. https ://doi.org/10.3109/10428191003793374.

24. Wawrzyniak E, Kotkowska A, Błoński JZ, Semeniuk-Ryś M, Ziółkowska E, Giannopoulos K, Robak T, Korycka-Wołowiec A. Clonal evolution In CLL patients as detected by FISH versus chromosome banding analysis, and its clinical significance. Eur J Haematol. 2013;92:91-101. https://doi.org/10.1111/ejh.12215.

25. Fegan C, Robinson H, Thompson P, Whittaker JA, White D. Karyotypic evolution in CLL: identification of a new sub-group of patients with deletions of $11 \mathrm{q}$ and advanced or progressive disease. Leukemia. 1995;9:2003-8.

26. Haferlach C, Dicker F, Schnittger S, Kern W, Haferlach T. Comprehensive genetic characterization of CLL a study on 506 cases analyzed with chromosome banding analysis, interphase FISH,
IGVH status and immunophenotyping. Leukemia. 2007;21:244251. https://doi.org/10.1038/sj.leu.2404935.

27. Baliakas P, Jeromin S, Iskas M, Puiggros A, Plevova K, NguyenKhac F, Davis Z, Rigolin GM, Visentin A, Xochelli A, Delgado J, Baran-Marszak F, Stalika E, Abrisqueta P, Durechova K, Papaioannou G, Eclache V, Dimou M, Iliakis T, Collado R, Doubek M, Calasanz MJ, Ruiz-Xiville N, Moreno C, Jarosova M, Leeksma AC, Panayiotidis P, Podgornik H, Cymbalista F, Anagnostopoulos A, Trentin L, Stavroyianni N, Davi F, Ghia P, Kater AP, Cuneo A, Pospisilova S, Espinet B, Athanasiadou A, Oscier D, Haferlach C, Stamatopoulos K, ERIC, the European Research Initiative on CLL. Cytogenetic complexity in chronic lymphocytic leukemia: definitions, associations, and clinical impact. Blood. 2019;133(11):1205-16. https://doi.org/10.1182/blood-2018-09873083.

28. Rigolin GM, del Giudice I, Formigaro L, Saccenti E, Martinelli S, Cavallari M, Lista E, Tammiso E, Volta E, Lupini L, Bassi C, Bardi A, Sofritti O, Daghia G, Cavazzini F, Marinelli M, Tavolaro S, Guarini A, Negrini M, Foà R, Cuneo A. Chromosome aberrations detected by conventional karyotyping using novel mitogens in chronic lymphocytic leukemia: clinical and biologic correlations. Genes Chromosomes Cancer. 2015;54:818-26. https://doi. org/10.1002/gcc.22293.

29. Janssens A, Van Roy N, Poppe B, Noens L, Philippé J, Speleman F, Offner F. High-risk clonal evolution in chronic B-lymphocytic leukemia: single-center interphase fluorescence in situ hybridization study and review of the literature. Eur J Haematol. 2012;89:72-80. https://doi.org/10.1111/j.1600-0609.2012.01790.

30. Stilgenbauer S, Sander S, Bullinger L, Benner A, Leupolt E, Winkler D, Kröber A, Kienle D, Lichter P, Döhner H. Clonal evolution in chronic lymphocytic leukemia: acquisition of high-risk genomic aberrations associated with unmutated $\mathrm{VH}$, resistance to therapy, and short survival. Haematologica. 2007;92:1242-5.

31. Loscertales J, Arranz E, Sanz MA, Blas C, Gil-Fernández JJ, Burgaleta C, Alegre A. Clonal evolution in patients with chronic lymphocytic leukemia. Leuk Lymphoma. 2010;51:1142-3. https ://doi.org/10.3109/10428191003793374.

32. Parikh SA, Strati P, Tsang M, West CP, Shanafelt TD. Should IGHV status and FISH testing be performed in all CLL patients at diagnosis? A systematic review and meta-analysis. Blood. 2016;127(14):1752-60. https://doi.org/10.1182/blood-2015-10620864.

33. Delgado J, Doubek M, Baumann T, Kotaskova J, Molica S, Mozas P, Rivas-Delgado A, Morabito F, Pospisilova S, Montserrat E. Chronic lymphocytic leukemia: a prognostic model comprising only two biomarkers (IGHV mutational status and FISH cytogenetics) separates patients with different outcome and simplifies the CLL-IPI. Am J Hematol. 2017;92:375-80. https://doi. org/10.1002/ajh.24660.

34. Rossi D, Fangazio M, Rasi S, Vaisitti T, Monti S, Cresta S, Chiaretti S, Del Giudice I, Fabbri G, Bruscaggin A, Spina V, Deambrogi C, Marinelli M, Famà R, Greco M, Daniele G, Forconi F, Gattei V, Bertoni F, Deaglio S, Pasqualucci L, Guarini A, DallaFavera R, Foà R, Gaidano G. Disruption of BICR1 associates with fludarabine chemorefractioriness in TP53 wild-type chronic lymphocytic leukemia. Blood. 2012;119:2854-62. https://doi. org/10.1182/blood-2011-12-395673.

35. Fabbri M, Bottoni A, Shimizu M, Spizzo R, Nicoloso MS, Rossi S, Barbarotto E, Cimmino A, Adair B, Wojcik SE, Valeri N, Calore F, Sampath D, Fanini F, Vannini I, Musuraca G, Dell'Aquila M, Alder H, Davuluri RV, Rassenti LZ, Negrini M, Nakamura T, Amadori D, Kay NE, Rai KR, Keating MJ, Kipps TJ, Calin GA, Croce CM. Association of a microRNA/TP53 feedback circuitry with pathogenesis and outcome of B-cell chronic lymphocytic leukemia. JAMA. 2011;305(1):59-67. https://doi.org/10.1001/ jama.2010.1919. 
36. Puente XS, Pinyol M, Quesada V, Conde L, Ordonez GR, Villamor N, Escaramis G, Jares P, Bea S, Gonzalez-Diaz M, Bassaganyas L, Baumann T, Juan M, Lopez-Guerra M, Colomer D, Tubio JM, Lopez C, Navarro A, Tornador C, Aymerich M, Rozman M, Hernandez JM, Puente DA, Freije JM, Velasco G, Gutierrez-Fernandez A, Costa D, Carrio A, Guijarro S, Enjuanes A, Herandez L, Yague J, Nicolas P, Romeo-Casabona CM, Himmelbauer H, Castillo E, Dohm JC, de Sanjose S, Piris MA, de Alava E, San Miguel J, Royo R, Gelpi JL, Torrentes D, Orozco M, Pisano DG, Valencia A, Guigo R, Bayes M, Heath S, Gut M, Klatt P, Marshall J, Raine K, Stebbings LA, Futreal PA, Stratton MR, Campbell PJ, Gut I, Lopez-Guillermo A, Estivill X, Monserrat E, Loez-Otin C, Campo E. Whole-genome sequencing identifies recurrent mutations in chronic lymphocytic leukaemia. Nature. 2011;475:101-5. https://doi.org/10.1038/nature10113.

37. Balatti V, Bottoni A, Palamarchuk A, Alder H, Rassenti LZ, Kipps TJ, Pekarsky Y, Croce CM. NOTCH1 mutations in CLL associated with trisomy 12. Blood. 2012;119:329-31. https://doi. org/10.1182/blood-2011-10-386144.

38. Roos-Weil D, Nguyen-Khac F, Chevret S, Touzeau C, Roux C, Lejeune J, Cosson A, Mathis S, Feugier P, Leprêtre S, Béné MC, Baron M, Raynaud S, Struski S, Eclache V, Sutton L, Lesty C, Merle-Béral H, Cymbalista F, Ysebaert L, Davi F, Leblond V, FILO working group. Mutational and cytogenetic analyses of 188 CLL patients with trisomy 12: a retrospective study from the French Innovative Leukemia Organization (FILO) working group. Genes Chromosomes Cancer. 2018;57:533-40. https://doi. org/10.1002/gcc.22650.

39. Oscier DG, Rose-Zerilli MJ, Winkelmann N, Gonzalez de Castro D, Gomez B, Forster J, Parker H, Parker A, Gardiner A, Collins A, Else M, Cross NC, Catovsky D, Strefford JC. The clinical significance of NOTCH1 and SF3B1 mutations in the UK LRF CLL4 trial. Blood. 2013;121:468-75. https://doi.org/10.1182/ blood-2012-05-429282.

40. Landau DA, Tausch E, Taylor-Weiner AN, Stewart C, Reiter JG, Bahlo J, Kluth S, Bozic I, Lawrence M, Böttcher S, Carter SL, Cibulskis K, Mertens D, Sougnez CL, Rosenberg M, Hess JM, Edelmann J, Kless S, Kneba M, Ritgen M, Fink A, Fischer K, Gabriel S, Lander ES, Nowak MA, Döhner H, Hallek M, Neuberg D, Getz G, Stilgenbauer S, Wu CJ. Mutations driving CLL and their evolution in progression and relapse. Nature. 2015;526:52530. https://doi.org/10.1038/nature15395.
41. Puente XS, Beà S, Valdés-Mas R, Villamor N, Gutiérrez-Abril J, Martín-Subero JI, Munar M, Rubio-Pérez C, Jares P, Aymerich M, Baumann T, Beekman R, Belver L, Carrio A, Castellano G, Clot G, Colado E, Colomer D, Costa D, Delgado J, Enjuanes A, Estivill X, Ferrando AA, Gelpí JL, González B, González S, González M, Gut M, Hernández-Rivas JM, López-Guerra M, Martín-García D, Navarro A, Nicolás P, Orozco M, Payer ÁR, Pinyol M, Pisano DG, Puente DA, Queirós AC, Quesada V, Romeo-Casabona CM, Royo C, Royo R, Rozman M, Russiñol N, Salaverría I, Stamatopoulos K, Stunnenberg HG, Tamborero D, Terol MJ, Valencia A, LópezBigas N, Torrents D, Gut I, López-Guillermo A, López-Otín C, Campo E. Non-coding recurrent mutations in chronic lymphocytic leukaemia. Nature. 2015;526:519-24. https://doi.org/10.1038/ nature14666.

42. Finn WG, Kay NE, Kroft SH, Church S, Peterson LC. Secondary abnormalities of chromosome $6 \mathrm{q}$ in B-cell chronic lymphocytic leukemia: a sequential study of karyotypic instability in 51 patients. Am J Hematol. 1998;59:223-9.

43. López C, Delgado J, Costa D, Villamor N, Navarro A, Cazorla M, Gómez C, Arias A, Muñoz C, Cabezas S, Baumann T, Rozman M, Aymerich M, Colomer D, Pereira A, Cobo F, López-Guillermo A, Campo E, Carrió A. Clonal evolution in chronic lymphocytic leukemia: analysis of correlations with IGHV mutational status, NOTCH1 mutations and clinical significance. Genes Chromosomes Cancer. 2013;52:920-7. https://doi.org/10.1002/gcc.22087.

44. Shanafelt TD, Jenkins G, Call TG, Zent CS, Slager S, Bowen DA, Schwager S, Hanson CA, Jelinek DF, Kay NE. Validation of a new prognostic index for patients with chronic lymphocytic leukemia. Cancer. 2009;10:24004. https://doi.org/10.1002/cncr.24004.

45. Rodríguez-Vicente AE, Bikos V, Hernández-Sánchez M, Malcikova J, Hernández-Rivas JM, Pospisilova S. Next-generation sequencing in chronic lymphocytic leukemia: recent findings and new horizons. Oncotarget. 2017;8:71234-48. https://doi. org/10.18632/oncotarget.19525.

Publisher's Note Springer Nature remains neutral with regard to jurisdictional claims in published maps and institutional affiliations. 